\title{
Unidad de dolor torácico: primera experiencia en Chile
}

\author{
Pablo Castro, Ramón Corbalán, Rodrigo Isa, Luigi G abrielli, \\ O svaldo Pérez, G astón Chamorro, Bernardita Garayar, \\ Ricardo Baeza, Ismael Vergara, Iván G odoy, Mónica Acevedo, \\ Alejandro Fajuri, Marcelo Fernández, José Miguel Mardones, \\ Alex Bittner, José Antonio Rodríguez.
}

\section{Chest pain unit: first experience in Chile}

Background: In large series, nearly $60 \%$ of admissions for suspected acute coronary syndrome (ACS) had a non-coronary etiology of the pain. However, short term mortality of non recognized ACS patients, mistakenly discharged from the emergency room is at least twice greater than the expected if they would had been admitted. The concept of a chest pain unit (CPU) is a methodological approach developed to address these issues. Aim: To evaluate the efficacy of a CPU in the emergency room of a general hospital for evaluation of acute chest pain. Material and Methods: Prospective study of patients with chest pain admitted in the CPU. After a clinical, electrocardiographic and laboratory evaluation with cardiac injury serum markers, patients were stratified in three risk groups, based on the likelihood of ACS of the American Heart Association. High probability patients were admitted to the Coronary Unit (CU) for treatment. Moderate probability patients remained in the CPU for further evaluation and low probability patients were discharged with telephonic follow-up. Results: Of 407 patients, 35, 30 and 35\% were stratified as high, intermediate and low probability ACS, respectively. Among patients admitted with high probability, $73 \%$ had a confirmed ACS diagnosis. Among intermediate probability patients, $86 \%$ were discharged after an evaluation in the CPU without adverse events in the follow-up. Conclusion: Structured risk evaluation approach in a CPU improves the management of acute chest pain, identifying high probability patients for fast admission and start of treatment in a $\mathrm{CU}$ and allowing safe discharge of low probability ones (Rev Méd Chile 2007; 135: 839-45).

(Key words: Bronchogenic cyst; Pancreatic neoplasms; Retroperitoneal neoplasms)

Recibido el 3 de octubre, 2006. Aceptado el 4 de enero, 2007.

Departamento de Enfermedades Cardiovasculares, Hospital Clínico y Facultad de Medicina, Pontifica Universidad Católica de Chile.

$\mathrm{E}^{\mathrm{s}}$ manejo de los pacientes que consultan en los servicios de urgencia (SU) por dolor torácico (DT) plantea un importante problema asistencial.

Correspondencia a: Dr. Pablo Castro G. Marcoleta 367, $6^{\circ}$ piso. Teléfono: 3543334. Fax: 6392037. E mail: pcastro@med.puc.cl
El DT representa entre $5 \%$ y $20 \%$ de las consultas en los SU de los hospitales generales y en $50 \%$ de los casos el cuadro clínico permite sospechar la presencia de un síndrome coronario agudo (SCA). No obstante, en menos de la mitad de los pacientes se confirma el diagnóstico ${ }^{1,2}$. Por otro 
lado, entre $2 \%$ y $10 \%$ de los pacientes que son dados de alta de los SU por considerar que el origen del dolor no es coronario, presentan un infarto agudo al miocardio, con una mortalidad que se duplica en relación a los enfermos ingresados en su primera evaluación ${ }^{3}$. Errores de este tipo constituyen entre $20 \%$ y $39 \%$ de los motivos de demandas judiciales en Estados Unidos de Norteamérica ${ }^{4}$ (EE.UU.). Por estas razones, la conducta de los médicos puede llegar a ser muy precavida, lo que se traduce en hospitalizaciones innecesarias y elevados costos.

Para mejorar la efectividad en el diagnóstico del DT en el SU se han propuesto diferentes soluciones, incluyendo el uso de guías de diagnóstico, la formación de equipos multidisciplinarios y el ingreso de estos pacientes en áreas específicas ${ }^{5-7}$. Esta última solución se conoce con el nombre de unidades de dolor torácico (UDT).

Estas unidades se crearon a partir de 1980 en EE.UU. con el objetivo primordial de reducir los tiempos de reperfusión coronaria en pacientes con infarto agudo al miocardio ${ }^{8}$. Con posterioridad, y ante la necesidad de mejorar la relación costoeficacia en los pacientes de bajo riesgo, que son los más frecuentes, se ampliaron los objetivos generales de estas unidades. Estos objetivos pasaron a ser la detección temprana y efectiva del síndrome coronario agudo, con los objetivos de diferenciarlo de otros procesos graves no coronarios (tromboembolismo pulmonar, disección aórtica, etc.), realizar una corecta estratificación del riesgo, identificar rápida y eficientemente los pacientes de bajo riesgo que pueden ser tratados en forma ambulatoria y, por último, realizar todo este proceso en el menor tiempo posible, disminuyendo los costos generales en la atención de estos pacientes. Actualmente, disponemos de los resultados del funcionamiento de estas UDT de centros hospitalarios en diferentes lugares del mundo ${ }^{1,8-10}$.

En agosto de 2002 creamos nuestra UDT, ubicada en el SU de nuestro hospital, dirigida por el Departamento de Enfermedades Cardiovasculares y coordinada con el SU, destinada a mejorar la atención del DT en un hospital terciario que atiende alrededor de 10.000 consultas anuales en el SU.

En el presente trabajo analizamos la estimación de probabilidad de un SCA y su correlación con el diagnóstico definitivo, la concordancia entre el diagnóstico de ingreso y egreso de los pacientes hospitalizados, el impacto de la obser- vación en la UDT sobre la conducta frente a un SCA dudoso y el seguimiento de los pacientes enviados a su domicilio desde el SU, directamente o luego de un período de observación.

\section{PACIENTES Y MÉTODO}

Estudio prospectivo de pacientes que consultaron por DT no traumático en el SU del Hospital Clínico de la Pontificia Universidad Católica de Chile (HCPUC) en horario diumo, en el periodo comprendido entre agosto 2002 y agosto 2003. La UDT fue organizada dentro del propio SU. El personal a cargo incluía una primera evaluación por un médico becado en cardiología junto a un cardiólogo de la planta médica, apoyado por enfermeras y auxiliares de enfermenía entrenadas en el reconocimiento oportuno del dolor coronario, interpretación básica de electrocardiograma (ECG) y fundamentos de terapia y reanimación cardiovascular.

De acuerdo a la historia clínica y a un ECG de reposo de doce derivaciones, los pacientes fueron clasificados en 4 categorías: SCA con supradesnivel del segmento ST (sdst) o BCRI, SCA sin sdst (s/sdst) definitivo, SCA s/sdst probable y dolor torácico no coronario. Los pacientes con SCA s/sdst definitivo, correspondían a aquellos que según clasificación de la American Heart Association tenían alta probabilidad de que su sintomatología fuese secundaria a un SCA, en tanto los de la categoría probable, correspondían a los pacientes de probabilidad intermedia o baja de tener un SCA (Tabla 1).

Otros procedimientos: Cuando el ECG era normal o no diagnóstico y el dolor era típico o atípico con factores de riesgo coronario presentes, se repetía un nuevo ECG a los 20 min y se determinaba troponina I. De ser estas negativas y aún no transcurrían más de $6 \mathrm{~h}$ del DT, el paciente entraba a un periodo de observación y se repetían las troponinas a las $6 \mathrm{~h}$ de la primera muestra. Cuando el ECG era normal o no diagnóstico y el dolor impresionaba claramente no coronario, se podían solicitar otros exámenes que estaban a criterio del cardiólogo evaluador (por ej: radiografía de tórax, scanner de tórax, otros).

Luego de la evaluación inicial y la clasificación del paciente, se procedió a una de las siguientes conductas: hospitalización, egreso al domicilio $\mathrm{u}$ otro sector del servicio de ungencia según lo 
determinara el médico responsable del paciente, $\mathrm{u}$ observación en la UDT.

En todos los pacientes hospitalizados se registró el diagnóstico definitivo al alta. En todos los pacientes enviados a su domicilio directamente desde el SU y aquellos enviados luego de la observación, se realizó un seguimiento telefónico a 30 días respecto a la necesidad de nueva consulta en servicio de urgencia, hospitalización y muerte del paciente (Figura 1).

Tabla 1. Probabilidad de que los signos y síntomas representen un síndrome coronario agudo secundario a enfermedad coronaria

\begin{tabular}{|llll|}
\hline \multirow{4}{*}{ Alta } & Probabilidad de síndrome coronario agudo \\
& Intermedia & Baja \\
\hline Historia & $\begin{array}{l}\text { Dolor típico con } \\
\text { episodios previos } \\
\text { documentados }\end{array}$ & $\begin{array}{l}\text { Dolor típico } \\
\text { Mayor de 70 años }\end{array}$ & $\begin{array}{l}\text { Dolor o síntomas } \\
\text { atípicos }\end{array}$ \\
& Sexo masculino & Uso de cocaína \\
& DM & \\
Examen & Insuficiencia mitral & Enfermedad & Dolor que se \\
& Hipotensión & vascular periférica & reproduce con la \\
& Diaforesis & & palpación \\
Edema pulmonar & & Normal \\
ECG & $\begin{array}{l}\text { Desnivel de ST u } \\
\text { onda T con síntomas }\end{array}$ & Onda Q & ST o T anormal \\
Marcadores & Elevados & Normal & Normal \\
\hline
\end{tabular}

Adaptado de Braunwald E. y col. Unstable angina: diagnosis and management. NHLBI 1994.

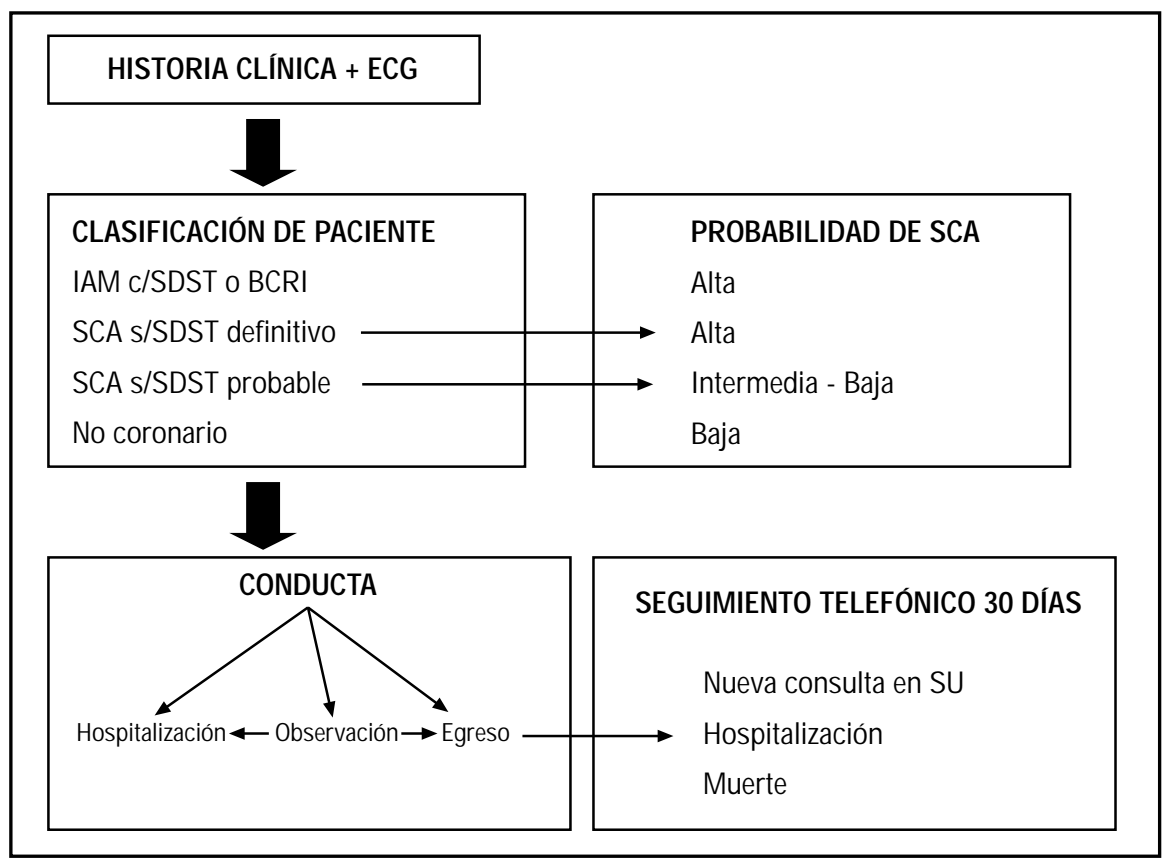

Figura 1. Protocolo de actuación ante un dolor torácico sospechoso de SCA (ver texto). 


\section{RESULTADOS}

En el período estudiado se registraron 9.246 consultas en el SU-HCPUC, correspondiendo 4,4\% (407 pacientes) a consultas por DT en horario diurno y atendidas por un cardiólogo.

Las características generales del grupo se muestran en la Tabla 2. La edad promedio de los pacientes fue de $58 \pm 14$ años y $69 \%$ eran hombres. La prevalencia de factores de riesgo coronario fue: hipertensión arterial en 41\%, tabaquismo en $37 \%$, dislipidemia en $36 \%$ y diabetes mellitus en $13 \%$.

De acuerdo a la clasificación de Braunwald y cols de probabilidad de tener un SCA luego de la evaluación clínica y ECG, 35\% de los pacientes tuvo alta probabilidad de SCA, 30\% tuvo probabilidad intermedia y 35\% tuvo baja probabilidad.

El $59 \%$ de los pacientes fue hospitalizado directamente, luego de la evaluación inicial, 21\% fue observado en la UDT y $20 \%$ fue enviado a su domicilio u otro sector del SU.

En los pacientes clasificados como de alta probabilidad de un SCA, se confirmó el diagnóstico definitivo en $98 \%$ de los casos, en aquellos de probabilidad intermedia, la confirmación diagnóstica fue de $52 \%$ y en los de baja probabilidad fue de 14\% (Figura 2). Del total de pacientes hospitalizados con diagnóstico de SCA se confirmó el diagnóstico en el 73\% de los casos.

El tiempo promedio de observación en la UDT fue de $108 \pm 62 \mathrm{~min}$. La conducta final en estos
Tabla 2. C aracterísticas de los pacientes evaluados en la unidad de dolor torácico

\begin{tabular}{|lrr|}
\hline & $\mathrm{N}^{\circ}$ & $\%$ \\
\hline Consultas en SU & 9.246 & \\
Consultas DT & 407 & 4,4 \\
& & \\
Edad & $58 \pm 14$ & \\
Hombres & 281 & 69 \\
Mujeres & 126 & 31 \\
Factores de riesgo & & \\
$\quad$ Hipertensión arterial & 166 & 41 \\
Tabaquismo & 151 & 37 \\
Dislipidemia & 148 & 36 \\
$\quad$ Diabetes mellitus & 53 & 13 \\
Probabilidad de SCA & & \\
$\quad$ Alta & 139 & 35 \\
Intermedia & 117 & 30 \\
$\quad$ Baja & 139 & 35 \\
Conducta inicial & & \\
$\quad$ Hospitalización & 238 & 59 \\
Observación & 86 & 21 \\
$\quad$ Egreso & 83 & 20 \\
\hline
\end{tabular}

pacientes fue la hospitalización en $14 \%$ de los casos y el egreso a su domicilio en $86 \%$. El seguimiento telefónico a los 15 días se efectuó en 95\% de los pacientes enviados a su domicilio, ya sea directa-

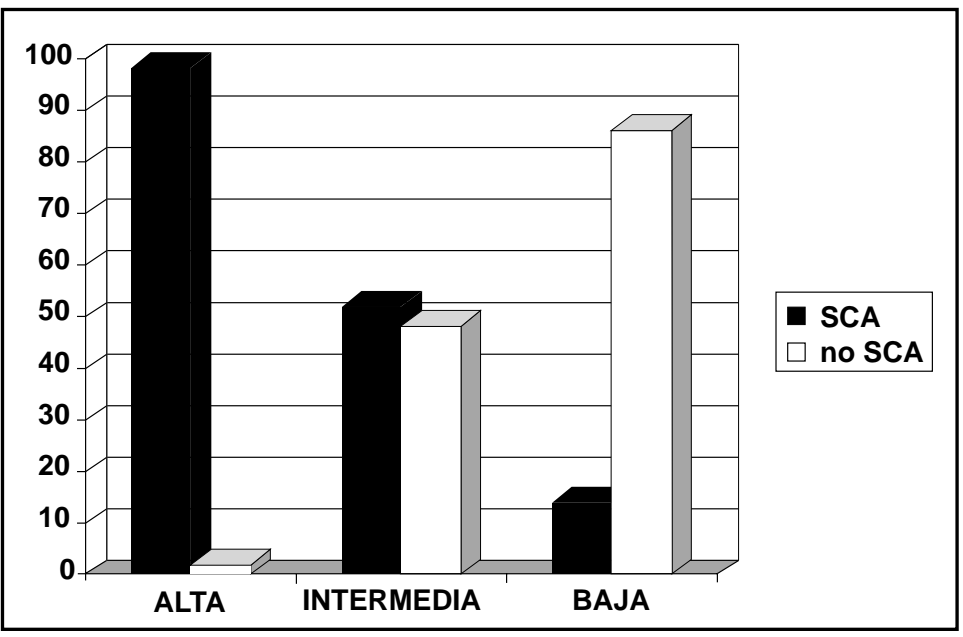

Figura 2. Comelación entre la probabilidad de tener un SCA estimada en la primera evaluación y el diagnóstico definitivo. 
mente luego de la evaluación inicial o luego de observación en la UDT, no registrándose eventos cardiovasculares o muerte en ninguno de ellos.

\section{DisCUSIÓN}

Nuestro estudio confirma que el dolor torácico es un motivo de consulta importante en los servicios de urgencia lo cual justifica contar con protocolos de manejo adecuado. Estos protocolos permiten diagnosticar en forma oportuna a los pacientes que cursan con un síndrome coronario agudo $\mathrm{u}$ otras causas graves de dolor torácico.

De acuerdo a nuestros resultados, la aplicación de las normas de la AHA para estimar la probabilidad de un síndrome coronario agudo fue de utilidad en la estratificación de los pacientes con dolor torácico en el servicio de urgencia, existiendo buena correlación con el diagnóstico definitivo y la decisión de ingreso u egreso hospitalario. Es así como sólo en $27 \%$ de los pacientes ingresados con diagnóstico presunto de síndrome coronario agudo no se confirmó el diagnóstico. Si bien en este grupo de pacientes la hospitalización podría haberse evitado, este porcentaje se compara favorablemente con la experiencia publicada ${ }^{9}$.

En este sentido, el contar con un área destinada a la observación de pacientes en los cuales el diagnóstico es dudoso, permitió que una gran proporción de los pacientes observados fueran enviados a su domicilio, lo que obviamente contribuye a optimizar los costos de la atención de salud.

Al mismo tiempo, no registramos eventos clínicos en los pacientes enviados a su domicilio. Es necesario considerar que el dolor torácico puede constituir un problema médico legal. Es así como en distintas experiencias se ha comunicado que un porcentaje alto de pacientes dados de alta desde el servicio de urgencia pueden presentar eventos cardiovasculares serios, con nuevas consultas en los servicios de urgencia y eventualmente mortalidad $^{11}$. La interpretación errónea de la causa de dolor torácico en los servicios de urgencia constituye entre $20 \%$ y $30 \%$ de demandas judiciales en los servicios de urgencia de EE.UU. Esta situación se da especialmente en síndromes coronarios agudos de pacientes más jóvenes ${ }^{4,12}$.
En los últimos años se han propuesto diversas soluciones para mejorar el diagnóstico del dolor torácico en los servicios de urgencia. Ello ha pasado por la implementación de pautas de práctica clínica, la formación de equipos multidisciplinarios y la evaluación de los pacientes en una unidad de dolor torácico. En esta se pueden aplicar distintas estrategias de acuerdo a los recursos de cada hospital. Es así como en algunos centros se han diseñado estrategias de evaluación complementaria precoz con técnicas no invasivas en todos aquellos casos en que la causa del dolor torácico es de origen dudoso. Estas pueden ir desde una prueba de esfuerzo convencional o con radioisótopos. Estos criterios se basan en el alto valor pronóstico de una prueba de esfuerzo negativa. Si consideramos, sin embargo, que los pacientes estudiados con técnicas no invasivas complementarias tienen baja probabilidad de enfermedad coronaria la probabilidad de resultados falsos positivos puede ser alta ${ }^{13-16}$. Por otra parte, es necesario evaluar los costos adicionales que implica la utilización de estas estrategias de estratificación de riesgo y el tiempo adicional que implican. Por último es necesario considerar el eventual riesgo de efectuar pruebas de provocación de isquemia en pacientes que pueden tener otras causas graves de dolor torácico como motivo de consulta. Por ello estimamos que las pruebas de provocación de isquemia debieran efectuarse en diferido en aquellos casos de dolor torácico de origen dudoso, aunque este criterio sea discordante con las pautas recomendadas en países desarrollados y con mayores recursos económicos ${ }^{17-19}$. Respecto a esto último, las normas norteamericanas de manejo del dolor torácico en el servicio de urgencia, recomiendan el uso precoz de la prueba de esfuerzo convencional o con radioisótopos dependiendo de la disponibilidad y experiencia de cada centro. Sobre el uso de la ecocardiografía convencional y con estrés, su utilidad en el contexto de la unidad de dolor torácico no es clara $^{20}$. Sin embargo, la ecocardiografía y otros estudios con imágenes han mostrado utilidad en el diagnóstico diferencial, en especial, con el síndrome aórtico agudo ${ }^{21}$. Por otro lado, la ecocardiografía con estrés tendría un rol en pacientes considerados de riesgo bajo para descartar cuadros coronarios agudos, evitando hospitalizaciones y estudios angiográficos innecesarios ${ }^{22}$. 
En nuestra experiencia, basándonos en criterios fundamentalmente clínicos, apoyados con la determinación de enzimas marcadoras de daño miocárdico, logramos tiempos de evaluación cortos en los casos de dolor torácico e interpretación incierta y logramos resultados satisfactorios. Así, sólo el 27\% de los casos ingresados con diagnóstico presunto de síndrome coronario agudo no se confirmó el diagnóstico y entre los pacientes enviados a domicilio no registramos eventos clínicos de importancia. Estos resultados preliminares nos llevan a plantear la importancia de un equipo de trabajo multidisciplinario, liderado por un cardiólogo, para el diagnóstico y manejo del dolor torácico en los servicios de urgencia de hospitales tipo A. Ello posibilita, además, no sólo el diagnóstico precoz de los síndromes coronarios agudos dudosos, sino también el de los infartos del miocardio, casos en que el tiempo que transcurre entre el diagnóstico y la toma de decisiones es crítico.

Entre las limitaciones de este estudio es necesario considerar que esta muestra, relativamente pequeña, se limita al análisis de aquellos pacientes que consultaron en horario diurno. Debemos

\section{REFERENCIAS}

1. Farkoun MF, Smars PA, Reeder GS, Zinsmeister AR, Evans RN, Meloy TD et AL. A clinical trial of a chest-pain observation unit for patients with unstable angina. Chest Pain Evaluation in the Emergency Room (CHEER) Investigators. N Engl J Med 1998; 339: 1882-8.

2. Arós F, Loma-Osorio A. Diagnosis of unstable angina in the emergency room. The clinical value and limitations of electrocardiography and other tests. Rev Esp Cardiol 1999; 52 Suppl 1: 39-45.

3. Lee TH, Rouan GW, Weisberg MC, Brand DA, Acampora D, Stasiulewicz et AL. Clinical characteristics and natural history of patients with acute myocardial infarction sent home from the emergency room. Am J Cardiol 1987; 60: 219-24.

4. Storrow AB, Gilber WB. Chest pain centers: diagnosis of acute coronary syndromes. Ann Emerg Med 2000; 35: 449-61.

5. Bayón J, Alegría E, Bosh X, Genover X, Cabadés A, IgLESIAS I ET AL. Chest pain units. Organization and señalar que los criterios de manejo del dolor torácico en horario nocturno en nuestro servicio de urgencia son similares. Al mismo tiempo ésta es una muestra representativa de pacientes que consultan por dolor torácico en un servicio de ungencia, correspondiendo a $4,7 \%$ de las consultas, cifras comparables a otras de la literatura ${ }^{5}$.

En resumen esta experiencia preliminar permitió conocer la magnitud del DT en nuestro medio, muestra que con personal con entrenamiento clínico adecuado, protocolos de manejo, electrocardiografía y algunos elementos básicos de laboratorio se puede estratificar en forma adecuada a los pacientes que consultan por esta causa en los servicios de urgencia. Además, este protocolo se asoció a una baja proporción de hospitalizaciones innecesarias y permitió enviar en forma segura a pacientes con baja probabilidad de enfermedad coronaria a su domicilio. Cabe resaltar finalmente que para la implementación de una unidad de dolor torácico lo más importante es la buena coordinación y voluntad entre los médicos responsables del SU y los cardiólogos del equipo evaluador.

protocol for the diagnosis of acute coronary syndromes. Rev Esp Cardiol 2002; 55: 143-54.

6. LEwis WR, Amsterdam EA. Defining the role of chest pain units. J Am Coll Cardiol 2001; 37: 2050-2.

7. Zalenski RJ, SelKer HP, Cannon CP, Farin HM, Gibler WB, GoldBerg RJ ET AL. National Heart Attack Alert Program position paper: chest pain centers and programs for the evaluation of acute cardiac ischemia. Ann Emerg Med 2000; 35: 462-71.

8. Pastor Torres LF, Pavon-Jiménez R, Reina Sánchez M. Chest pain unit: one-year follow-up. Rev Esp Cardiol 2002; 55: 1021-7.

9. Steve Goodacre, Jon Nichoц, Simon Dixon. Randomized controlled trial and economic evaluation of a chest pain observation unit compared with routine care. BMJ 2004; 328: 254-60.

10. Bassan R, GilbLer WB. Chest pain units: state of the art of the management of patients with chest pain in the emergency department. Rev Esp Cardiol 2001; 54: 1103-9.

11. Pope JH, Aufderheide TP, Ruthazer R, Woolard RH, Feldman JA, Beshansky JR et al. Missed diagnoses 
of acute cardiac ischemia in the emergency department. N Engl J Med 2000; 342: 1163-70.

12. Rusnak RA, Stair TO, Hansen K, Fastow JS. Litigation against the emergency physician: common features in cases of missed myocardial infarction. Ann Emerg Med 1989; 18: 1029-34.

13. Stein R, Chaitman B, Balady J, Fieq JL, Limacher MC, PINa IL ET AL. Safety and utility of exercise testing in emergency room chest pain centers: An advisory from the Committee on Exercise, Rehabilitation, and Prevention, Council on Clinical Cardiology, American Heart Association. Circulation 2000; 102: 1463-7.

14. Sanchis J, Bodi V, Luacer A, Nunez J, Ferrero JA, CHORRo FJ ET AL. Value of early exercise stress testing in a chest pain unit protocol. Rev Esp Cardiol 2002; 55: 1089-92.

15. Conti A, Zanobetti M, Grifoni S, Berni G, Costanzo E, GAWNI C ET AL. Implementation of myocardial perfusion imaging in the early triage of patients with suspected acute coronary syndromes. Nucl Med Commun 2003; 24: 1055-60.

16. Gómez MA, Anderson JL, Karagounis LA, Muhlestein JB, MoozRs FB. An emergency department-based protocol for rapidly ruling out myocardial ischemia reduces hospital time and expense: results of a randomized study (ROMIO). J Am Coll Cardiol 1996; 28: 25-33.

17. Braunwald E, Antman EM, Beasley JW, Califf RM, Cheitun MD, Hochman JS ET AL. ACC/AHA guidelines for the management of patients with unstable angina and non-ST-segment elevation myocardial infarction: executive summary and recommendations. A report of the American College of Cardiology/American Heart Association task force on practice guidelines (committee on the management of patients with unstable angina). Circulation 2000; 102: 1193-209.

18. Arós F, Loma-Osorio A, Alonso A, Alonso JJ, Cabades A, Coma-Canela I et al. The clinical management guidelines of the Sociedad Espanola de Cardiologia in acute myocardial infarct. Rev Esp Cardiol 1999; 52: 919-56.

19. American Colegge of Emergency Physicians. Clinical policy: critical issues in the evaluation and management of adult patients presenting with suspected acute myocardial infarction or unstable angina. American College of Emergency Physicians. Ann Emerg Med 2000; 35: 521-5.

20. Hutter AM JR, Amsterdam EA, JaFFe AS. $31^{\text {st }}$ Bethesda Conference. Emergency Cardiac Care. Task force 2: Acute coronary syndromes: Section 2B-Chest discomfort evaluation in the hospital. J Am Coll Cardiol 2000; 35: 853-62.

21. KaUL S. Doppler echocardiography in critically ill cardiac patients. Cardiol Clin 1991; 9: 711-32.

22. Conti A, Sammicheli L, Gauni C, Constanzo EN, Antoniucci D, Barletta G. Assessment of patients with low-risk chest pain in the emergency department: Head-to-head comparison of exercise stress echocardiography and exercise myocardial SPECT. Am Heart J 2005; 149: 894-901. 